\title{
Designing Smart Particles for the Assembly of Complex Macroscopic Structures**
}

\author{
Esther Garcia-Tunon, * Suelen Barg, Robert Bell, Jonathan V. M. Weaver', Claudia Walter, \\ Lidia Goyos, and Eduardo Saiz.
}

In memory of Jon Weaver

The development of modern technologies, from tissue engineering to catalysis or energy storage, requires novel lightweight structures combining optimum mechanical and functional response. ${ }^{[1-4]}$ This demand is driving the development of new manufacturing techniques to build materials with complex shapes and architectures exhibiting characteristic features from the nanoscopic level and up. Ideally, these techniques should be flexible, remain low-cost and enable high-volume fabrication. A very attractive alternative is the use of wet processing technologies based on smart particles that are able to assemble in response to external stimuli. Ideally, the assembly process should be reversible and programmable, allowing an effective manipulation of the strength of particle-particle interactions. However, the design of responsive particles to build complex meso- to macroscopic structures starting from assembly at the nano- to microscale is very challenging, and today its use is limited mostly to nanostructures and coatings. ${ }^{[5,6]}$

Herein we describe a novel manufacturing process to fabricate macroscopic inorganic structures with complex architectures based on the design of responsive particle surfaces. The method uses a $\mathrm{pH}$-responsive branched copolymer surfactant (BCS $)^{[7-9]}$ to functionalize the surfaces in situ and create smart inorganic particles that can self-disperse or assemble between themselves or with soft templates (that is, in emulsified suspensions) under the action of an external trigger $(\mathrm{pH})$. The process allows the fabrication of strong materials with complex shapes, and a wide range of micro- to macroscale architectures from dense to foams with closed or open cells or even graded structures (Figure 1). The method does not depend on the particle chemistry but on the interactions between the BCS molecules functionalizing the

[*] Dr. E. Garcia-Tunon, Dr. S. Barg, R. Bell, Dr. J. V. M. Weaver, Dr. C. Walter, L. Goyos, Prof. E. Saiz

Department of Materials, Imperial College London (UK)

E-mail: esther.garcia-tunon@imperial.ac.uk

[†] Deceased.

$[* *$ ] The authors would like to thank the EPSRC Science and Innovation grant Building New Capability in Structural Ceramics (EP/F033605/ 1), the European Commission (FP7-Marie Curie Intra-European Fellowship and International Reintegration Grants), the Pedro Barrie de la Maza Foundation, and the National Institute of Health (NIH, Grant No. 1 R01 DE015633) for financial support.

Supporting information for this article, including experimental details, specific examples of the ceramic structures, and results of mechanical characterization, is available on the WWW under http:// dx.doi.org/10.1002/anie.201301636.

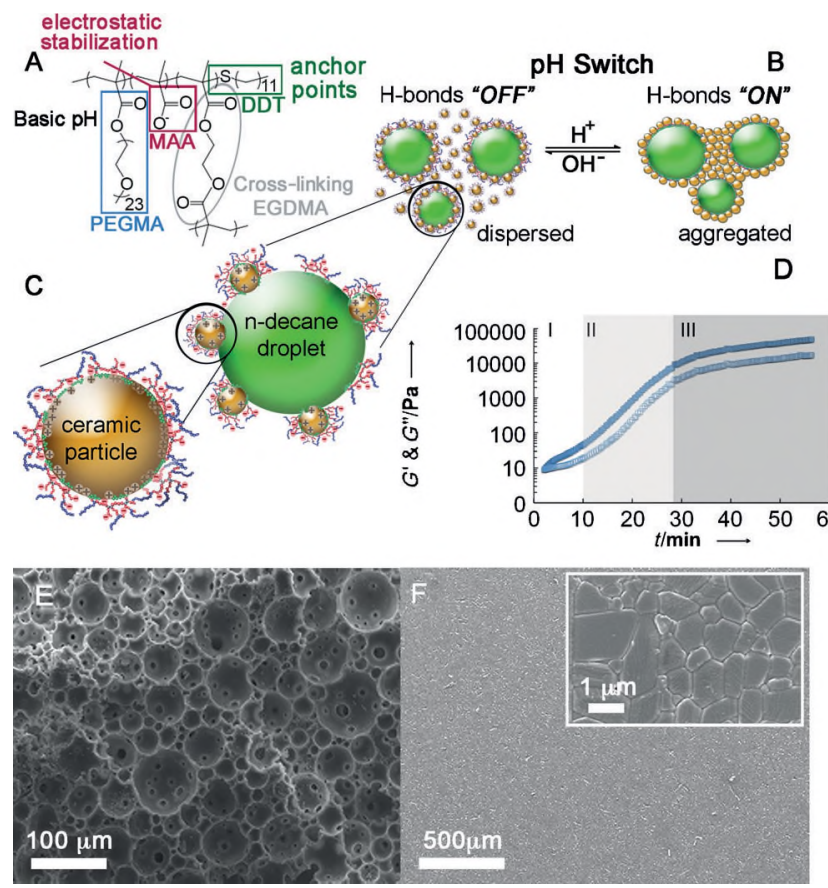

Figure 1. A) BCS structure showing the branch functionalities. B) $\mathrm{pH}$ triggered assembly of an oil-in-water emulsified suspension of BCSfunctionalized ceramic particles. C) The BCS-particle-droplet and BCS-particle interactions. D) Evolution of the viscoelastic $\left(\mathrm{G}^{\prime}, \mathrm{G}^{\prime \prime}\right)$ properties of an emulsified suspension with changing $\mathrm{pH}$, illustrating the assembly process. Particles and droplets are initially steric and electrostatically stabilized at high $\mathrm{pH}$, the MAA branches are in their anionic form, and the hydrogen bonds are switched off. When the $\mathrm{pH}$ value drops below 5 (longer times), the MA branches are completely protonated (hydrogen bonds switched on) and the functionalized particles and droplets (B) bond, forming a network; $\mathrm{G}^{\prime}$ (filled symbols) and $\mathrm{G}^{\prime \prime}$ (open symbols) reach values above $20 \mathrm{kPa}$. E,F) Examples of ceramic structures fabricated through responsive self-assembly:

E) porous $\mathrm{SiC}$ from an emulsion and F) sintered highly dense (>99\% of the theoretical value) alumina obtained from a suspension (fourpoint bending strength, $200 \pm 50 \mathrm{MPa}$ ).

surfaces, and herein we use $\mathrm{Al}_{2} \mathrm{O}_{3}$ and $\mathrm{SiC}$ to illustrate its versatility.

The BCS is based on methyl methacrylic acid (MAA) and polyethylene glycol methacrylate (PEGMA) with hydrophobic dodecanethiol (DDT) chain ends and ethylene glycol di-methacrylate (EGDMA) as cross linker (Figure 1 A). ${ }^{[7-9]}$ This amphiphilic molecule can segregate to oil-water interfaces and stabilize them. ${ }^{[9]}$ Furthermore, the branched architecture ensures that each molecule contains multiple poten- 
tial points of attachment to the surface of an inorganic particle to functionalize it and promote its segregation to oil-water interfaces ${ }^{[10]}$ in an emulsion (Figure 1B,C). Attachment and functionalization occurs by the following mechanisms: 1) The interactions of the hydrophobic chain ends (DDT) on the surfaces; 2) the electrostatic interaction between the carboxylic anions in the MAA residues $\left(\mathrm{COO}^{-}\right)$with the positively charged particle surfaces; and 3) the establishment of chemical covalent bonding between the carboxylic residues and the metal oxides on the surface of the particles (Figure 1). ${ }^{[1,12]}$

The process starts with the BCS functionalization of the inorganic particles in concentrated water-based suspensions (for details, see the Supporting Information). Analysis of these suspensions by dynamic light scattering (DLS) and inducted coupled spectroscopy (ICP) shows that for particles with sizes of the order of $200-500 \mathrm{~nm}$ the optimum BCS concentration ranges between 1 and $2 \% \mathrm{wt} / \mathrm{v}$ (with regards to volume of suspension). At these BCS concentrations the particles are well-dispersed, and ICP analyses of the sulfur concentrations in the liquid indicate that $26 \%$ of BCS molecules are attached to the particles, while $74 \%$ remain in solution. At basic $\mathrm{pH}(8-12)$, the functionalities in the branches (EG and MAA) provide steric and electrostatic stabilization, allowing the formulation of well-dispersed colloidal suspensions that can reach very high solid contents (up to $60 \mathrm{vol} \%$ ). If needed for the fabrication of complex structures, these colloidal suspensions can be easily emulsified with decane (50-60 vol \% related to the suspension volume). The oil droplets are stabilized by a synergetic combination of the BCS molecules in the liquid and the functionalized particles, both of which attach to the oil-water interface (Figure 1B,C). Particles with wetting angles of the order of $70^{\circ}$ attach irreversibly to the oil-water interface with higher energy than usually observed for surfactant molecules. ${ }^{[13,14]}$ In this respect, functionalization with BCS modifies the wettability of the ceramic particle surfaces increasing the water contact angle (Supporting Information, Figure S3). ${ }^{[15-18]}$ Particle-BCS stabilized emulsions can be stored without destabilization for more than 1 month. These stable droplets act as a temporary soft template for the fabrication of porous structures (Figure 1E).

At high $\mathrm{pH}$, both suspensions and emulsions are very fluid (with viscosities between 0.02-0.04 Pa). However, under acidic conditions (below the apparent $\mathrm{p} K_{\mathrm{a}}$ of the MAA residues, $\mathrm{p} K_{\mathrm{a}} \approx 5$ ) the BCS-functionalized surfaces "turn on" multiple links between the molecules, functionalizing particles and droplets as the neutral MAA residues form hydrogen bonds with the EG residues (Figure 1B). In this way, a $\mathrm{pH}$ switch triggers a self-assembly process to form in situ solid parts that can be easily handled and retain the complex shape of the mold. Subsequently these parts can be dried and sintered to form fully inorganic structures that can be dense (from suspensions) or porous (from emulsions) with a wide range of architectures depending on the degree of emulsification, the properties of the starting suspension, the kinetics of the self-assembly and the solvent evaporation rate. The variables that affect the kinetics and strength of the assembly are temperature, relative concentration of BCS, solid loading, and amount of the $\mathrm{pH}$ regulator (herein we used glucono- $\delta$-lactone, G $\delta \mathrm{L}$, the hydrolysis of which to gluconic acid is used to homogeneously decrease in $\mathrm{pH}$ ).

As the $\mathrm{pH}$ decreases, the dispersed-to-aggregated phase transition can be divided in three stages: I) early stage assembly, II) network formation, and III) plateau (Figure $1 \mathrm{D})$. During the early stage, a weak gel network is established; the crossover point that indicates gel formation (where the viscoelastic properties, storage $\left(\mathrm{G}^{\prime}\right)$ and loss $\left(\mathrm{G}^{\prime \prime}\right)$ moduli reach the same value) takes place shortly after triggering the $\mathrm{pH}$ drop. As the $\mathrm{pH}$ decreases, more links between particles and droplets are turned on and the gel becomes rapidly a stiff network (stage II, Figure 1D). During the third stage, the moduli reach a plateau where the formation of multiple hydrogen bonds creates a strong particle network. Larger BCS concentrations lead to longer aggregation times, which is due to its buffer role while higher particle concentrations result in the faster formation of stronger materials, as in diluted suspensions the long distance between particles results in slower aggregation and a weaker particle network. We can easily reverse the assembly process by switching off the hydrogen bonds in the networks by, for example, submerging the structures in alkaline aqueous solution ( $\mathrm{pH} 12$; Figure $2 \mathrm{~A}$ ). The structures disassemble into dispersed particles and droplets owing to hydrogen-

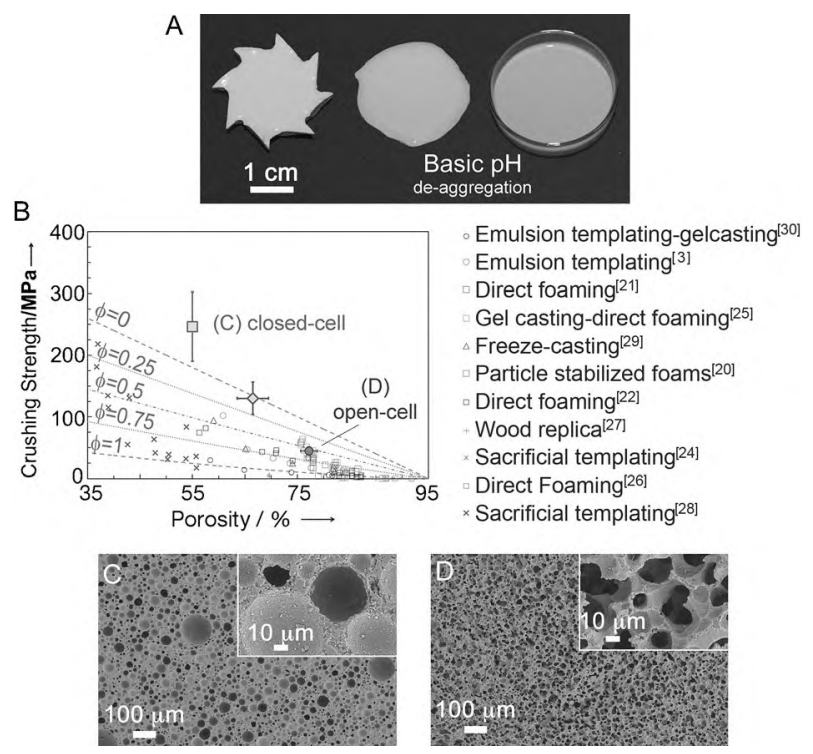

Figure 2. A) Reversible aggregation of a complex-shaped porous structure obtained by emulsion templating (left); de-aggregation of the particle/droplet network in alkaline water (middle); ceramic suspension recovered after "turning off" the hydrogen bonds (right). B) Compressive strength of the cellular $\mathrm{Al}_{2} \mathrm{O}_{3}$ ceramics synthesized herein compared with reported data for porous alumina foams ${ }^{[17,19-30]}$ The theoretical predictions from the Gibson and Ashby model are included for comparison (dashed lines; considering a three-point bending strength of $400 \mathrm{MPa}$ for dense counterparts; $\phi$ is the fraction of solids in cell faces, $0=$ closed cell, $1=$ open cell). ${ }^{[31]}$ C),D) Corresponding microstructures: C) closed-cell $\mathrm{Al}_{2} \mathrm{O}_{3}$ (porosity ca. $55 \%$ ) obtained from a suspension containing $43 \mathrm{vol} \%$ of particles in BCS solution (1\% wt/ $\mathrm{v}, \mathrm{pH} 8$ ) emulsified with $50 \mathrm{vol} \%$ decane at $2000 \mathrm{rpm}$; D) alumina structure with highly interconnected porosity (porosity ca. $80 \%$ ) obtained from a suspension containing $15 \mathrm{vol} \%$ of particles homogenized with $50 \mathrm{vol} \%$ decane at $24000 \mathrm{rpm}$. Higher emulsification speeds lead to a finer interconnected porosity. 
bond decomplexation and electrostatic repulsion of the anionic MAA residues on the droplet and particle surfaces.

The self-assembly of particles and soft templates provides an excellent route to tune the architecture of cellular ceramics. By manipulating the emulsification conditions (solid content of the starting suspension, amount of decane used to emulsify and emulsification speed; see the Supporting Information) and the relative kinetics of self-assembly and drying, we can manufacture cellular ceramics with complex shapes and a broad range of microstructures ranging from closed-cell to highly interconnected open porosity (Figure $2 \mathrm{C}, \mathrm{D})$ or structures exhibiting graded architectures, from dense to highly porous (Figure 3 ). For example, we

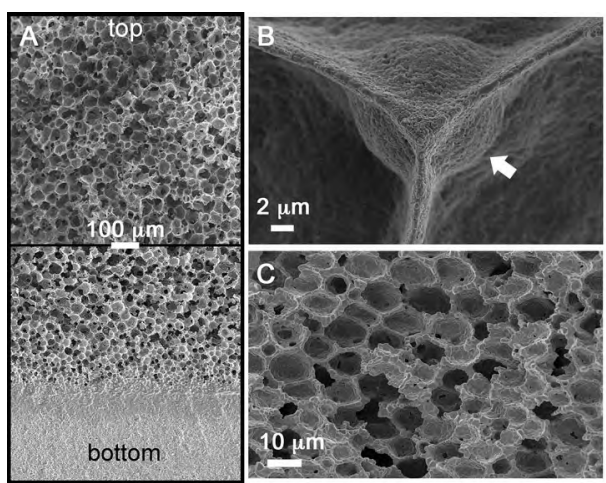

Figure 3. A) SEM image of a sample with graded porosity. B),C) Structure formed from a 12 vol\% alumina suspension emulsified with 50 vol \% of decane and subjected to slow-rate solvent elimination: B) Detail of a strut between adjacent pores formed following the plateau border between decane droplets. The sample has not yet been sintered and the walls between pores resulting from the emulsion lamellae are about $500 \mathrm{~nm}$ thick. The bubble behind the strut (arrow) illustrates the efficient packing of droplets. C) Honeycomb structure obtained after sintering the material in (B).

have prepared closed-cell cellular ceramics with 50-60\% porosity (average size $30 \mu \mathrm{m}$ ), and a proportion of open pores below $1-2 \%$ or homogeneous and highly porous cellular materials with $80-90 \%$ porosity (average size $15 \mu \mathrm{m}$ with a narrow size distribution between 9 and $30 \mu \mathrm{m}$ ) in which up to $72 \%$ is interconnected and open (Figure 2).

These porous materials exhibit high compressive strengths that in the case of close-cell ceramics can be more than double of those previously reported for similar macroporous ceramics $^{[17,19-30]}$ and could be even above the predictions of the Gibson and Ashby model ${ }^{[31]}$ (Figure 2B). This extraordinary mechanical performance reflects the meso- to macroscale structure of the materials and the efficiency of the process. The low viscosity, stability, and wide droplet size distribution of the emulsions (from $10 \mu \mathrm{m}$ to $80 \mu \mathrm{m}$ ) allow an efficient packing of the oil droplets before assembly and the formation of a remarkably homogeneous 3D architecture. As a consequence, the final pores are not monodisperse, which may explain the deviations from the Gibson and Ashby model. ${ }^{[31]}$ Moreover, the establishment of a strong particle network through the assembly process combined with the very low organic content of the suspension and the use of a soft template help to preserve the integrity of the inorganic part during drying and sintering and enables the formation of dense, defect-free walls (Figure 2C).

Graded porous structures (Figure $3 \mathrm{~A}$ ) can be created by emulsifying suspensions with low solid loading (7-15 vol\%) at moderate stirring speeds $(2000 \mathrm{rpm})$ to create emulsions with limited stability and wide droplet size distributions. The destabilization leads to the formation of a dense layer at the bottom while the oil droplets arrange hierarchically above it, owing to the different densities of oil and water. By tuning the assembly and destabilization kinetics, for example by using relatively high amounts of GoL (12-15\% wt/v), it is possible to freeze this hierarchical arrangement (small and stable droplets at the bottom and larger and less stable droplets at the top) to form scaffolds with graded porosity (Figure $3 \mathrm{~A}$ ). Moreover, the pore shape of highly porous scaffolds can also be controlled from spherical to polyhedral (Figure 3B,C) by manipulating the solvent evaporation rate. Long drying times enable lamella formation between adjacent oil droplets (Figure 3B), which leads to characteristic polyhedral shapes and highly porous (70-80\%) cellular ceramics with very thin walls $(\leq 1 \mu \mathrm{m})$ after sintering (Supporting Information, Figure S4).

In summary, we have formulated a new approach for the synthesis of smart particles that are able to assemble in response to an external stimulus. These particles can be used in the bottom-up manufacture macroscopic inorganic structures with complex shapes and architectures. The method is independent of the particle chemistry, is versatile, reversible and simple, using surface functionalization with small additions of a single polymer to enable dispersion and trigger assembly. The rheological response of the suspensions can be manipulated to support a range of manufacturing techniques including the formulation of injectable ceramic inks for solid free-form fabrication or injection molding (Supporting Information, Figure S4). The process addresses two standing issues in the fabrication of highly porous materials: how to reliably form them into complex shapes, and how to increase strength, opening new paths for the fabrication of lightweight structural components. The potential for application is very broad including light filters, ceramic films with graded porosity, bulk thermal shock-resistant structures, thermal barrier coatings, or temperature control membranes for automatic thermal reforming, to mention a few. In particular, current research in our group is focusing on the use of closed-cell ceramics as lightweight thermal insulators with structural capabilities and the application of open cell structures as catalytic supports and tissue engineering scaffolds.

[1] P. Colombo, Philos. Trans. R. Soc. A 2006, 364, 109-124.

[2] A. R. Boccaccini, M. Erol, W. J. Stark, D. Mohn, Z. Hong, J. F. Mano, Compos. Sci. Technol. 2010, 70, 1764-1776. 
[3] J. Hüppmeier, S. Barg, M. Baune, D. Koch, G. Grathwohl, Fuel 2010, $89,1257-1264$.

[4] A. Tampieri, G. Celotti, S. Sprio, A. Delcogliano, Biomaterials 2001, 22, 1365-1370.

[5] M. Grzelczak, J. Vermant, E. M. Furst, L. M. Liz-Marzán, ACS Nano 2010, 4, 3591-3605.

[6] M. A. C. Stuart, W. T. S. Huck, J. Genzer, M. Müller, C. Ober, M Stamm, G. B. Sukhorukov, I. Szleifer, V. V. Tsukruk, M. Urban, F. Winnik, S. Zauscher, I. Luzinov, S. Minko, Nat. Mater. 2010, 9, $101-113$.

[7] J. V. M. Weaver, S. P. Rannard, A. I. Cooper, Angew. Chem. 2009, 121, 2165-2168; Angew. Chem. Int. Ed. 2009, 48, 2131 2134.

[8] R. T. Woodward, L. Chen, D. J. Adams, J. V. M. Weaver, J. Mater. Chem. 2010, 20, 5228-5234.

[9] R. T. Woodward, J. V. M. Weaver, Polym. Chem. 2011, 2, $403-$ 410.

[10] J. Weaver, D. J. Adams, Soft Matter 2010, 6, 2575-2582.

[11] S. Bertazzo, K. Rezwan, Langmuir 2010, 26, 3364-3371.

[12] M. E. Karaman, D. A. Antelmi, R. M. Pashley, Colloids Surf. A 2001, 182, 285-298.

[13] P. N. Sturzenegger, U. T. Gonzenbach, S. Koltzenburg, L. J. Gauckler, Soft Matter 2012, 8, 7471-7479.

[14] G. Kaptay, Colloids Surf. A 2006, 282-283, 387-401.

[15] I. Akartuna, A. R. Studart, E. Tervoort, U. T. Gonzenbach, Langmuir 2008, 24, 7161-7168.

[16] B. P. Binks, Curr. Opin. Colloid Interface Sci. 2002, 7, 21-41.
[17] U. T. Gonzenbach, A. R. Studart, E. Tervoort, L. J. Gauckler, Angew. Chem. 2006, 118, 3606-3610; Angew. Chem. Int. Ed. 2006, $45,3526-3530$.

[18] S. U. Pickering, J. Chem. Soc. 1907, CXCVI, 2001-2021.

[19] S. Barg, Cellular Ceramics via Alkane Phase Emulsified Powder Suspensions, Shaker, Aachen (Germany), 2010.

[20] U. T. Gonzenbach, A. R. Studart, D. Steinlin, E. Tervoort, L. J. Gauckler, J. Am. Ceram. Soc. 2007, 90, 3407-3414.

[21] S. Barg, D. Koch, G. Grathwohl, J. Am. Ceram. Soc. 2009, 92, $2854-2860$.

[22] S. Dhara, P. Bhargava, Int. J. Appl. Ceram. Technol. 2006, 3, $382-$ 392.

[23] Y. S. Han, J. B. Li, Y. J. Chen, Mater. Res. Bull. 2003, 38, $373-$ 379.

[24] Y. S. Han, J. B. Li, Q. M. Wei, K. Tang, Ceram. Int. 2002, 28, 755 759

[25] X. Mao, S. Shimai, S. Wang, J. Eur. Ceram. Soc. 2008, 28, $217-$ 222

[26] H. X. Peng, Z. Fan, J. Evans, Ceram. Int. 2000, 26, 887-895.

[27] C. R. Rambo, H. Sieber, Adv. Mater. 2005, 17, 1088-1091.

[28] E. Ryshkewitch, J. Am. Ceram. Soc. 1953, 36, 65-68.

[29] B. H. Yoon, W. Y. Choi, H. E. Kim, J. H. Kim, Y. H. Koh, Scr. Mater. 2008, 58, 537-540.

[30] B. Yuan, H. Wu, X. Sun, G. Wang, H. Li, Mater. Lett. 2012, 81, $151-154$.

[31] L. J. Gibson, M. F. Ashby, Cellular Solids: Structure and Properties, Cambridge University Press, Cambrige, 1999. 